Personality Psychopathology: Longitudinal Prediction of Change

in BMI and Weight Post-Bariatric Surgery

Joshua R. Oltmanns (MS)1, Jessica Rivera Rivera (MS)1, Jonathan Cole (PhD, ABPP)2,3, Amanda Merchant (PhD, ABPP)2,3, and Joshua P. Steiner (MD, FACS)3

1University of Kentucky, 2Bluegrass Health Psychology, 3St. Joseph's Center for Weight Loss Surgery

(C) 2019, American Psychological Association. This paper is not the copy of record and may not exactly replicate the final, authoritative version of the article. Please do not copy or cite without authors' permission. The final article will be available, upon publication, via its DOI: 10.1037/hea0000842

Authors'note:

Joshua R. Oltmanns, Department of Psychology, University of Kentucky; Jessica Rivera Rivera, Department of Psychology, University of Kentucky; Jonathan Cole, Bluegrass Health Psychology; Amanda Merchant, Bluegrass Health Psychology; Joshua P. Steiner, St. Joseph’s Center for Weight Loss Surgery.

This research was supported by the National Institute of Aging under Award Number F31AG055233. The content is solely the responsibility of the authors and does not necessarily represent the official views of the National Institutes of Health.

Correspondence should be addressed to Joshua R. Oltmanns, Department of Psychology, University of Kentucky, 111-D Kastle Hall, Lexington, KY, 40506. Email: jroltmanns@uky.edu 


\begin{abstract}
Objective: Bariatric surgery is an effective treatment for obesity, which has been increasing worldwide. However, bariatric surgery causes dramatic physical changes that can cause significant stress. Prior research has found that psychological variables such as personality traits and levels of psychopathology can influence success after bariatric surgery (in terms of bodymass index $[\mathrm{BMI}]$ reduction and weight loss). However, most prior studies have been limited by small sample sizes, inconsistent follow-up, and categorical assessment of psychopathology.

Methods: The present study examines the predictive utility of the Personality Assessment Inventory (PAI) scales for three bariatric surgery outcomes (BMI reduction, weight loss, and percent excess weight loss [\%EWL]) across ten follow-up points five years after surgery. It also examines the largest sample of bariatric surgery-completing patients $(N=2,267)$ on the PAI to date. Latent growth modeling was used to examine change in the outcome variables. Results: Results indicate that personality and psychopathology variables predicted less BMI reduction, weight loss, and \%EWL five years after surgery and also affected the trajectories of change in the outcome variables across time. The PAI scales predicted more variance in the five-year BMI outcomes than did age and gender. The most robust effects were for scales assessing phobias, traumatic stress, identity problems, and negative relationships. Conclusions: The PAI may be useful to clinical health psychologists who conduct recommended psychological evaluations with potential bariatric surgery candidates.
\end{abstract}

Keywords: health psychology, bariatric surgery, personality, personality disorder, psychopathology 


\section{Personality Psychopathology: Longitudinal Prediction of Change in BMI and Weight Post-Bariatric Surgery}

Obesity is a significant worldwide public health concern and its prevalence has increased over the past few decades (Finucane et al., 2014). Bariatric surgery is a medical procedure designed to reduce obesity. It has become the most effective treatment for obesity by causing rapid and significant weight loss that can lead to partial and even complete remission of health problems such as diabetes, dyslipidemia, arterial hypertension, and sleep apnea (Adams et al., 2012; Aguera et al., 2015; Buchwald et al., 2004; Courcoulas et al., 2013; Puzziferri et al., 2014). The resulting physical changes following bariatric surgery are dramatic and require a lifelong post-operation nutritional regimen and sustained concentration on maintaining the health benefits of surgery. Enduring the physical changes and managing the required lifestyle changes can lead to stress (Karlsson Taft, Sjöström, Torgerson, \& Sullivan, 2003; White et al., 2015). Patients with pre-existing mental health problems may be less able to adapt to the physical and emotional stresses associated with bariatric surgery, which may lead to problems maintaining weight loss and additional psychosocial difficulties (Bhatti et al., 2016). As a result, The American Society for Metabolic and Bariatric Surgery recommends broad psychological testing before bariatric surgery (Sogg, Lauretti, \& West-Smith, 2016) and clinical health psychologists have an important role in evaluating patient potential for post-bariatric surgery success.

A significant amount of research has been conducted on the psychological factors that influence post-surgery weight loss (Marek, Ben-Porath, \& Heinberg, 2016; Wimmelmann, Dela, \& Morensen, 2014). Weight loss after bariatric surgery is correlated with improved quality of life (Brunault et al., 2015; Karlsson et al., 2003), while weight regain can undo the positive health benefits of bariatric surgery (Wimmelmann et al., 2014). Although there appear to be several 
psychological variables that predict weight loss after surgery, the findings have been inconsistent. Mood and anxiety disorder symptoms (Aguera et al., 2015; de Zwaan et al., 2011; Wimmelmann et al., 2014), binge eating disorder symptoms (Engel, Mitchell, de Zwaan, \& Steffen, 2012), and maladaptive and normal-range personality traits have predicted less weight loss after bariatric surgery (Gerlach, Herpertz, \& Loeber, 2015; Livhits et al., 2012). Specifically, maladaptive personality traits of neuroticism and disinhibition predict less weight loss (Bond, Phelan, Leahey, Hill, \& Wing, 2009; Canetti, Berry \& Elizur, 2009). In contrast, the adaptive trait of cooperativeness was found to enhance weight loss (Aguera et al., 2015). Personality may predict weight loss primarily through the mechanisms of eating behavior and/or stress generation (Wimmelmann et al., 2014). For example, having disinhibited personality traits may affect postsurgery control over eating habits. Similarly, more neurotic, anxious, and depressed patients may experience more stress, which may lead to weight gain through maladaptive eating behaviors or through the physiological effects of stress (O’Leary, 1990). However, many of these same psychological variables have been found not to significantly predict weight loss after bariatric surgery (e.g., mood and anxiety disorder symptoms, binge eating disorder symptoms, and personality traits; Herpertz, Kielmann, Wolf, Hebebrand, \& Senf, 2004; Kalarchian et al., 2016; Marek et al., 2016; Wimmelmann et al., 2014).

There are several reasons for the inconsistent findings in this literature (Marek et al., 2016; van Hout, Verschure, \& van Heck, 2005). Many studies have used relatively small samples of bariatric surgery patients-for example, one set of studies reviewed previously ranged in sample size from $N=35$ to $N=361$ (Wimmelmann et al., 2014). Smaller samples provide less statistical power to detect weight loss outcomes after bariatric surgery-especially when the effect sizes are not large (the effect sizes have been small-to-moderate; Marek et al., 2016; 
Wimmelmann et al., 2014). Another important methodological consideration is the length of time after bariatric surgery in which outcomes have been measured. Many studies examine outcomes only one-year post-surgery. This is important because most patients follow the same immediate and dramatic weight loss trajectory within the first year after surgery (Courcoulas et al., 2013). It appears that many psychological individual difference variables become important for weight loss trajectories after one year (Magro et al., 2008). Another important consideration is the measurement method of psychological variables. Some studies have used categorical variables to predict weight change (i.e., placing patients into yes/no diagnostic categories based on responses to psychological tests), while others have used dimensional scales to predict weight change (i.e., measuring psychological variables along a continuum). Dimensional measurement provides more statistical power to detect change through enhancing measurement reliability (Marek et al., 2016). Largely for this reason, the dimensional classification of personality and psychopathology is becoming increasingly promoted and utilized in the $21_{\text {st }}$ century (Kotov et al., 2017). Finally, another potential contributor to the inconsistency of prior findings is the reliance on dichotomous decisions about the predictive validity of psychosocial variables based solely on decisions using $p$-values. The prior literature may appear more consistent paying attention to effect sizes rather than only arbitrary thresholds of statistical significance (Wasserstein, Schirm, \& Lazar, 2019). The goal of the present study is to add robust findings to the literature by using a larger sample size, longer follow-up times, dimensional measurement of personality and psychopathology, and paying particular attention to effect sizes rather than solely p-values. These methodological improvements should help provide useful and dependable insight into psychological factors that affect post-bariatric surgery success. 
In order to uncover more reliable personality and psychopathology predictors of bodymass index (BMI) reduction and weight loss after bariatric surgery, higher powered studies will be necessary. One recent longitudinal study of BMI trajectories five years post-bariatric surgery used dimensional measurement of personality and psychopathology with a relatively large sample $(N=446)$. Marek Ben-Porath, van Dulmen, Ashton, and Heinberg (2017) examined whether dimensional scales of personality psychopathology from the Minnesota Multiphasic Personality Inventory-Restructured Form (Ben-Porath, 2012) predicted BMI trajectories over and above categorical psychopathology diagnoses. They used latent growth modeling (LGM; Bollen \& Curran, 2006; Grimm, Ram, \& Estabrook, 2017)—which is a more recently popularized and efficient form of structural equation modeling that is used specifically to examine change in variables across time - to examine the utility of the MMPI-2-RF scales in the prediction of BMI reduction. They found that the MMPI-2-RF Behavioral/Externalizing Dysfunction (BXD; including assessment of disinhibition, aggression, substance use, and borderline, antisocial, and narcissistic personality traits), Low Positive Emotions (assessing anhedonia, unhappiness, and suicidal tendencies), and Hypomanic Activation (assessing thought racing, impulsivity, aggression, and substance abuse) scales predicted higher BMI at the five-year outcome (i.e., these scales predicted the LGM intercept). The effects were small, but statistically significant ( $\beta$ s $=.11-.13)$, and were incremental over and above the effects of age and binge eating disorder. The results from this study also indicated that the Hypomanic Activation, Anger Proneness, and Activation scales predicted a slower rate of BMI reduction across time. In sum, this study provided strong supporting evidence that disinhibited and depressed personality and psychopathology traits predict less longitudinal BMI reduction five years after bariatric surgery. 
The present study examines data collected from a sample of 2,267 bariatric surgery patients across five years after surgery and uses the Personality Assessment Inventory (PAI; Morey, 2007), which is a widely used dimensional clinical measurement of personality and psychopathology (Piotrowski, 2017). The PAI is used in various clinical, forensic, research, and personnel settings. Several advantages of the instrument include 1) scales with common personality and psychopathology construct names (such as depression, schizophrenia, borderline, and antisocial personality scales), 2) focus on content and discriminant validity and reduction of item bias during its development, and 3) inclusion of several interpersonal and treatment consideration scales that can aid with client conceptualization, in addition to several wellsupported validity scales. To our knowledge, the present study is the largest sample of bariatric surgery-completing patients that has been examined with the PAI to date.

The present study examines weight, BMI, and percent excess weight loss (\%EWL) across 10 follow-up time points after bariatric surgery, with five time points after the first year (i.e., at 18 months, 24 months, 36 months, 48 months, and 60 months). This allowed outcome and change trajectories to be modeled during the crucial time frame when most individual differences in weight loss begin to appear (i.e., after the first year; Magro et al., 2008). The greater number of time points will allow for statistical modeling of more nuanced changes and variability in BMI reduction, weight loss, and \%EWL across time (Bollen \& Curran, 2006). Because of the inconsistent findings in this literature, the prediction analyses in the present study are exploratory. In addition to examining the predictive utility of personality and psychopathology in the prediction of weight loss outcomes, the effects of age, gender, and surgery type are examined, which have all been shown to affect weight trajectories after bariatric surgery. Gastric 
bypass surgery generally shows the greatest weight reduction in the years following surgery (Courcoulas et al., 2013).

In sum, the present study is, to our knowledge, the largest and highest-powered study to examine PAI prediction of BMI and weight trajectories after bariatric surgery. The predictive validity evidence of PAI scores, which will be useful for making clinical recommendation decisions about bariatric surgery candidates. The large sample of patients, time points, and latent growth modeling provides an opportunity to gain valuable information about patients who completed bariatric surgery, about bariatric surgery success indicators, and about the clinical health predictive utility of the PAI five years post-surgery.

\section{Method}

\section{Procedure}

Potential bariatric surgery candidates completed the PAI during psychological evaluations at an outpatient health psychology clinic. Approved surgery candidates underwent bariatric surgery at a local medical center for weight loss surgery. Patients $(N=2,267)$ underwent either Roux-en-Y gastric bypass surgery $(n=1,147)$, sleeve gastrectomy $(n=1,035)$, or gastric banding $(n=81)$ surgeries 1 . The majority of patients completed surgery between 2010 and $2018(n=2,154)$, with fewer between 2002-2009 ( $n=113)$. Patients completed follow-up appointments at the center for weight loss at 1 month $(n=2,123 ; 6 \%$ missing $), 3$ months $(n=$ 1,805; 20\% missing), 6 months ( $n=1,552 ; 31 \%$ missing), 9 months ( $n=352 ; 84 \%$ missing), 12 months ( $n=1,299 ; 34.5 \%$ missing), 18 months ( $n=320 ; 86 \%$ missing), 24 months $(n=620$; $73 \%$ missing), 36 months ( $n=328 ; 86 \%$ missing $), 48$ months $(n=228 ; 90 \%$ missing), and 60 months ( $n=163 ; 93 \%$ missing). 
Height and weight were measured and body-mass index (BMI) was calculated at each follow-up. Percent excess weight loss (i.e., percent of initial weight minus ideal weight [decided by life insurance guidelines] lost at each follow-up; \%EWL) was calculated at all follow-ups except for at 18 months. The three primary outcomes in the present study are BMI, weight loss, and $\%$ EWL across the time points. The present study was approved by the University of Kentucky Institutional Review Board (IRB protocol \#17-0796-P4S). Patients did not complete informed consent because the data were archival. The study's preregistration and data analysis syntax are available at: https://osf.io/u28ne/.

\section{Patients}

Patients were 2,267 adults (Mage $=48.6$ years, $S D=11.2$ years) who completed a psychological assessment, were recommended for surgery, and completed bariatric surgery. Patients were $76.4 \%$ female $(n=1,731)$ and primarily white $(76 \%)$ and black $(6.2 \%)$, with $4.8 \%$ identifying with multi or other racial backgrounds. They were primarily married $(60.5 \%)$, but also single (14.7\%), divorced (11.6\%), cohabiting (11.5\%), and widowed (1.7\%). Educational backgrounds were diverse: $16.8 \%$ completed less than 12 th grade, $22 \%$ completed 12 th grade, $28.4 \%$ completed less than four years post high school, $11.4 \%$ completed four years after high school, and $21.5 \%$ completed more than four years after high school. Descriptive BMI, weight, and \%EWL statistics for each follow-up are included in the supplemental materials.

\section{Personality Assessment Inventory}

Patients completed the 344-item PAI. Items were rated on a 4-point scale: not at all true, slightly true, mainly true, or very true. The PAI consists of eleven clinical scales that have corresponding subscales, five treatment scales, four validity scales, and two interpersonal scales. Clinical scales assess different forms of psychopathology such as anxiety, depression, and 
psychotic disorders; treatment scales assess constructs that may be influential in treatment such as aggression, suicidal ideation, and stress; validity scales assess potential responding styles such as random responding and impression management; and interpersonal scales assess dominant and warm interpersonal styles (Morey, 2007). The PAI has demonstrated strong construct validity and reliability in numerous studies since its creation (see Gardner, Boccaccini, Bitting, \& Edens, 2015; Patry \& Magaletta, 2015; Slavin-Mulford et al., 2012). T-scores produced by the PAI software were used in analyses. As recommended by Morey (2007) to screen for random responding, patients with scores of $>70$ on the Inconsistency scale or $>72$ on the Infrequency scale $(n=21)$ were removed from the dataset. No adjustments were made for positive or negative impression management, as these are often overlapping with meaningful individual differences (Kurtz et al., 2008; McCrae \& Costa, 1983).

\section{Statistical Analyses}

The data were first visualized for each outcome using ggplot2 (Wickham, 2016) in R statistical software (R Core Team, 2013). Latent growth models were fit using the lavaan R package (Rosseel, 2012). Models were fit for three outcomes: 1) BMI, 2) weight loss, and 3) \%EWL. Each model included three covariates: patient age, gender, and a dummy variable representing surgery type ( 1 for gastric bypass, for which the majority of patients received, and 0 for sleeve or band). The intercept was specified at the 60-month (five-year) time point. Linear models were fit first and then compared to quadratic models because prior studies indicate that weight loss after bariatric surgery follows a quadratic shape (Courcoulas et al., 2013; Marek et al., 2017).

Data collection for bariatric psychological assessments and outcome data post-surgery is continuous and ongoing — as a result, missing data increases at each time point. The present 
analyses utilize all available data using full information maximum likelihood (FIML) estimation. FIML takes into account all available data points to estimate trajectories and is a better alternative to imputing or excluding missing data (Enders \& Bandalos, 2001; Peugh \& Enders, 2004). In longitudinal studies, it is recommended that all available data be used, and sensitivity analyses be conducted between follow-up completers and non-completers (Newman, 2014): Using a pre-specified significance level of .001 (as is used throughout the study), patients who did not return for follow-up appointments post-bariatric surgery were compared with patients who did return for follow-up appointments on the PAI clinical, interpersonal, and treatment consideration scales using $t$-tests. There were no significant mean differences on these PAI scales between the two groups, nor did the two groups differ in mean initial BMI or initial weight. The correlation between initial BMI and number of missed follow-ups was $r=.01(p=.786)$ and the correlation between initial weight and number of missed follow-ups was $r=.03(p=.204)$, indicating little-to-no relations between BMI, weight, and completing follow-up appointments. As a result, it can be assumed that any bias in parameter estimates would be small (Newman, 2014).

Throughout the results section, standardized regression effects will be interpreted as small (<.3), moderate (between .30 and .50), and large (> .50; Cohen, 1992; Ferguson, 2009). As the prior literature is perhaps inconsistent due lack of power and the use of dichotomous decisions about predictive ability of variables using $p$ values alone (e.g., arbitrarily based on $p>$ or $<.05$ ), effect sizes are used to interpret the results (Wasserstein et al., 2019), while using a guideline of $p<.001$ for statistical significance.

\section{Results}

\section{Descriptive Statistics}


Descriptive statistics for the main PAI scales are displayed in Table 1 and for the PAI subscales in the supplemental materials (Table S1). Descriptive statistics for the PAI and the three outcome measures at each follow-up (by gender) are provided in Tables S2-S4 of the supplemental materials. Coefficient alpha for the main clinical, treatment consideration, and interpersonal scales ranged from $\alpha=.42$ (Drug Problems; DRG) to $\alpha=.90$ (Anxiety; ANX), with a median of $\alpha=.79$. Coefficient alpha for the subscales ranged from $\alpha=.36$ (Egocentricity) to $\alpha=.83$ (Traumatic Stress and Affective Depression), with a median of $\alpha=.70$. Bariatric patients scored higher on Somatic Complaints (SOM) than the normative PAI sample. Intercorrelations among the PAI scales are presented in supplemental materials Table S5. Figures S1-S3 of the supplemental materials display the five-year BMI, weight loss, and \%EWL trajectories for patients following bariatric surgery. Each outcome clearly displayed a quadratic growth trend, with BMI and weight showing a rapid reduction in the first months after surgery, followed by a slower reduction in the years after surgery. \%EWL displayed a rapid increase in the first months after surgery, followed by a slower increase in the years after surgery.

\section{Model Fit}

The model fit indices are presented in Table S6. It is evident that quadratic models with free loadings on the quadratic slope between the initial and 60-month time points fit the data best, meeting subjective thresholds for good model fit (e.g., CFI > .90, RMSEA $<.080$, and SRMR < .080; Kline, 2015). Freeing loadings is often useful for improving model fit in nonlinear growth models (Grimm et al., 2017). An example quadratic model is presented in Figure 1.

\section{Effects of Control Variables}


Effects of control variables in all three final models are presented in Table S7. Male gender predicted a higher five-year BMI, while gastric bypass surgery predicted a lower fiveyear BMI, holding all other variables constant. These effects were small and moderate, respectively. Age was not significantly associated with five-year BMI. However, older age was associated with a higher BMI linear slope value after surgery (i.e., a slower rate of BMI reduction) at a small effect size. Male gender and gastric bypass procedure were associated, in contrast, with lower linear slope values after surgery, at small effect sizes. There was a small negative effect of age on the quadratic slope, indicating that older age was associated with a slower quadratic acceleration rate in BMI reduction (i.e., older patients did not reduce BMI as rapidly as younger patients). There were small and moderate positive effects of male gender and bypass on the quadratic slope, respectively. Similar results were found for weight. However, in the weight models, the effects of male gender on the intercept and slopes were moderate.

In the \%EWL model, gastric bypass surgery predicted higher five-year \%EWL at a moderate effect size, holding all other variables constant, indicating that patients who underwent gastric bypass lost more excess weight across time. Age and male gender had small negative effects on the linear slope of \%EWL after surgery and gastric bypass surgery a small positive effect. Gastric bypass surgery had a moderate negative effect on the quadratic slope, indicating that gastric bypass patients displayed faster acceleration trajectories in \%EWL. The effects of age and male gender on the quadratic slope were positive and small.

\section{All PAI Scales Predicting Outcomes}

First, the incremental validity of all PAI scales together over the covariates was examined. Models including all of the PAI scales predicting the growth factors for BMI, weight, and $\%$ EWL fit the data significantly better than models including all of the PAI scales with their 
effects on the growth factors set to zero: $\chi_{2}(54)=194.53, \chi_{2}(54)=189.65$, and $\chi_{2}(54)=123.12$, respectively, all $p<.001$. It should be noted that a large amount of the explained variance $\left(R_{2}\right)$ in the growth factors was obviously attributable to the surgery type variable (i.e., gastric bypass was associated with more BMI reduction and weight loss), and in the weight model a large amount was attributable to gender. Thus, $R_{2}$ values were tested for the growth factors predicted only by age and gender in the BMI model. These values were $2 \%, 8 \%$, and $7 \%$ respectively for intercept, linear slope, and quadratic slope in the BMI model before adding the PAI scales. After adding the PAI scales as predictors of the BMI growth factors, standardized $R 2$ values for the intercept, linear slope, and quadratic slope increased to $6 \%, 12 \%$, and $11 \%$-increases in standardized $R_{2}$ of $4 \%, 6 \%$, and $4 \%$, respectively-over and above age and gender. This indicates that, as a whole, the PAI main scales predicted more variance in the five-year BMI outcome than age and gender and about half as much in the trajectory of change.

\section{Individual PAI Scales Predicting Outcomes}

Models were run separately to examine the predictive effects of each PAI scale in isolation. Results for BMI are displayed in Table 2. PAI scales generally positively predicted the five-year intercept, indicating that endorsement of the items on these scales was associated with a higher BMI five years after surgery. In particular, SOM, Anxiety-Related Disorders (ARD), Borderline Problems (BOR), Drug Problems (DRG), Stress (STR), Nonsupport (NON), Alcohol Problems (ALC), Paranoia (PAR), and Depression (DEP) had the largest relative effect sizes. The interpersonal scales had the smallest effect sizes. The unstandardized regression estimates can be interpreted as in linear regression (Bollen \& Curran, 2006): For example, a 1-unit increase in the ARD scale was associated with a .120-unit increase in the five-year BMI intercept, holding all other variables constant. The Treatment Rejection (RXR) scale predicted the intercept in the 
opposite direction, indicating that the higher the initial RXR score, the lower the five-year BMI outcome.

Regarding change trajectory, the PAI scales generally negatively predicted the linear slope, with the exceptions of RXR and the interpersonal scales, which were positive. In particular, the STR scale had the largest negative effect, indicating that a higher pre-surgery STR score was associated with slower BMI reduction across the study. The SOM and ARD scales also had relatively higher effect sizes. The RXR scale had the largest positive effect on the linear slope (indicating a faster reduction). The PAI scales generally positively predicted the quadratic slope (in particular the SOM and STR scales), indicating faster acceleration in BMI reduction after surgery. The RXR scale was negatively predictive of the quadratic slope, indicating a slower acceleration rate.

At the subscale level (Table 3), subscales from BOR, SOM, ARD, SCZ, PAR, DEP, and ANX, in particular ARD-Phobias (ARD-P) and Traumatic Stress (ARD-T), SOM Conversion (SOM-C), BOR Identity Problems and Negative Relationships (BOR-I and N, respectively), and SCZ Psychotic Experiences (SCZ-P), positively predicted the five-year BMI intercept at small effect sizes. Subscales again generally negatively predicted the linear slope, in particular subscales from SOM, BOR, ARD, and DEP. Prediction of the quadratic slope was again generally positive, indicating faster quadratic acceleration rates, with subscales from SOM, ARD, and DEP having the relatively largest effects.

Results of the models predicting change in weight are displayed in Table S8 and S9. PAI predictors were similar: Effects on the intercept were generally positive, negative on the linear slope and positive on the quadratic slope, with the exception of RXR, which was always in the opposite direction. In particular, ARD, STR, DRG, BOR, and NON had the largest relative 
effects on the five-year intercept. At the subscale level, again ARD-P, BOR-I, BOR-N, and SOM-C had some of the largest individual effects on the intercept. Results of the models predicting change in \%EWL are displayed in Table S10 and S11. In particular, ARD and DRG had the largest relative effects on the intercept and linear slope values, indicating higher values on these scales were associated with less \%EWL and slower change in \%EWL.

\section{Discussion}

Bariatric surgery is an effective treatment for obesity (Courcoulas et al., 2013). Despite its effectiveness, it may cause significant stress related to life-long changes in behavioral and dietary regimens. Psychological testing is therefore recommended before bariatric surgery (Sogg et al., 2016). The PAI is one of the most used and well-validated self-report measures of personality and psychopathology and can be used in psychological assessments of potential bariatric surgery patients. The present study examined its validity in the prediction of BMI reduction, weight loss, and \%EWL across five years after bariatric surgery.

Results from the present study indicate that personality and psychopathology variables add significant incremental variance to the prediction of five-year BMI, weight, and \%EWL change trajectories and outcomes over and above age, gender, and initial levels of the outcome variables. In particular, the effects of anxiety-related disorders, borderline personality problems, and stress predicted higher five-year outcomes in both BMI and weight after bariatric surgery. More specifically, within anxiety-related disorders and borderline personality problems, analyses at the subscale level showed that phobias and traumatic stress (from anxiety-related disorders) and identity problems and negative relationships (from borderline personality problems), respectively, were associated both with less optimal BMI and weight outcomes and less \%EWL across the five years. These effects were over and above initial levels of outcome variables, age, 
gender, and bariatric surgery type. Findings indicate that patients with fears and past traumatic stressful events, problems with self and identity, and rocky and unstable interpersonal relationships may have more difficulty with maintaining weight loss, BMI reduction and \%EWL in the five years following bariatric surgery.

The effects in the present study are interpreted as small effects, similar in magnitude to those found by Marek et al. (2017). For the PAI scales that predicted BMI, a one-point increase was — on average - equal to slightly more than one tenth of a BMI point across the five years controlling for the effects of age, gender, and surgery type. For the PAI scales that predicted higher weight, a one-point increase was — on average — equal to slightly more than one half of one pound controlling for the effects of prior weight, age, gender, and surgery type. For patients with higher scores on these variables, these effects may add up — that is, patients may have several elevated PAI scales. Indeed, PAI scale elevations tend to co-occur (see Table S5). However, these effects are likely underestimated because candidates were screened for psychopathology by licensed clinical health psychologists before receiving bariatric surgery, likely reducing the levels of psychopathology in the sample. Pre-surgical screening for psychopathology, in combination with any potential positive impression management that may have been present, and the relatively larger rate of missing data at the later outcomes, all would have worked to reduce the estimates of the effects (Newman, 2014). In sum, it is possible that these effect sizes are conservative.

Research indicates that mental health treatment after bariatric surgery influences outcome (Kalarchian \& Marcus, 2018; Robinson et al., 2014; Rydén et al., 2001). One study found that people with mental disorders who engaged in treatment had better outcomes than people with mental disorders who did not engage in treatment and people without mental disorders who did 
not engage in treatment (Shen, Lin, Huang, \& Yen, 2016). These findings, in combination with those from the present study, would indicate that personality and psychopathology variables can predict less optimal outcomes after bariatric surgery, but that if people with mental health disorders engage in treatment, they can benefit from bariatric surgery. Thus, psychological treatment should likely be encouraged to patients with mental health disorders before and after bariatric surgery — if mental health concerns are not deemed unsafe for bariatric surgery—and also perhaps to patients without mental health disorders.

Our results help put demographic predictors of weight loss into context. There has been inconsistency in prior studies, specifically, with regard to the predictive utility of age (Wimmelmann et al., 2014). The present results indicate that age does predict the trajectory of weight loss at a small effect size, with older patients displaying slower BMI and weight reduction across time (c.f., Marek et al., 2017). However, the present results indicate that personality traits — more so than age — predict the final five-year weight loss outcome over and above the age of the patient. The present results indicate that if age is an important factor in the prediction of weight loss after bariatric surgery, so too are personality and psychopathology.

There were several unexpected findings in the present study. First, treatment rejection (RXR) was consistently associated with better bariatric surgery outcomes. This scale measures an individual's self-reported need for treatment (Morey, 2007). The effects likely indicate that patients without histories of mental health treatment benefit more after bariatric surgery in BMI and weight reduction. Indeed, the RXR variable was negatively correlated with all but one of the other PAI scales (Table S5). Clinicians might then use the RXR scale as a positive predictor of bariatric surgery outcomes. It was also unexpected that STR and SOM predicted faster acceleration in BMI reduction. The likely explanation for these counter-intuitive findings is that 
patients with higher STR and SOM levels had higher initial BMI levels and patients with higher RXR had lower initial BMI levels. Indeed, the STR and SOM variables were two of five PAI scales that had significant correlations with initial BMI $(r$ 's $=.12$ and .14 , respectively, $p<.001$; these were among the strongest) and RXR was the only one with a negative correlation with initial BMI $(r=-.12, p<.001)$. Thus, there was more BMI for patients high on STR and SOM to lose and less BMI for patients high on RXR to lose in the beginning of the study-however, patients with higher STR and SOM ended up with slower BMI reduction overall (i.e., lower slope values) and higher BMIs across five years (i.e., higher intercept values).

\section{Limitations}

The present study, although providing a significant advance in terms of methodology, had some limitations. Due to the nature of the data collection process (i.e., collected continuously in a hospital setting across many years), there were higher levels of missing data at the later followups in the present study than in others (Marek et al., 2017). However, the present study examined a significantly larger sample and larger number of follow-ups than prior studies, it is recommended that researchers use all available data, and sensitivity analyses indicated no significant differences in terms of PAI, BMI, or weight scores were found between patients who completed the five years and those who did not. This indicates that patient-level missing data would be unlikely to meaningfully bias the parameters found in our results (Newman, 2014). Second, this study only included patients who received bariatric surgery after being screened for significant psychopathology. That, along with the potential for positive impression management during assessment, could potentially have influenced the results by reducing the coefficient estimates. Future studies should provide information about patients who are not recommended for bariatric surgery due to psychological assessments. 


\section{Conclusions}

Bariatric surgery is an important procedure because of its ability to help people with obesity. However, it causes dramatic physical changes that can take a psychological toll. Results from the present study provide a high-powered test of the ability of personality and psychopathology variables to indicate who may have more difficulty after bariatric surgery across several years. Findings indicate that personality and psychopathology variables have important relationships with bariatric surgery outcomes. It appears potentially beneficial for mental health professionals to continue providing psychological assessments for potential bariatric surgery patients before surgery is completed, taking into account score on omnibus psychological inventories such as the PAI. The use of well-validated measures such as the PAI and the MMPI-2-RF may provide important aid to clinicians in identifying patients that may be at risk for problematic outcomes after bariatric surgery. 


\section{References}

Ben-Porath, Y. S. (2012). Interpreting the MMPI-2-RF. University of Minnesota Press.

Bhatti, J. A., Nathens, A. B., Thiruchelvam, D., Grantcharov, T., Goldstein, B. I., \& Redelmeier, D. A. (2016). Self-harm emergencies after bariatric surgery: a population-based cohort study. JAMA Surgery, 151, 226-232.

Bollen, K. A., \& Curran, P. J. (2006). Latent curve models: A structural equation perspective. Hoboken, NJ: Wiley.

Bond, D. S., Phelan, S., Leahey, T. M., Hill, J. O., \& Wing, R. R. (2009). Weight-loss maintenance in successful weight losers: surgical vs non-surgical methods. International Journal of Obesity, 33, 173-180.

Canetti, L., Berry, E. M., \& Elizur, Y. (2009). Psychosocial predictors of weight loss and psychological adjustment following bariatric surgery and a weight-loss program: The mediating role of emotional eating. International Journal of Eating Disorders, 42, 109117.

Cohen, J. (1992). A power primer. Psychological Bulletin, 112, 155-159.

Corsica, J. A., Azarbad, L., McGill, K., Wool, L., \& Hood, M. (2010). The Personality Assessment Inventory: Clinical utility, psychometric properties, and normative data for bariatric surgery candidates. Obesity Surgery, 20, 722-731.

Courcoulas, A. P., Christian, N. J., Belle, S. H., Berk, P. D., Flum, D. R., Garcia, L., ... \& Patterson, E. J. (2013). Weight change and health outcomes at 3 years after bariatric surgery among individuals with severe obesity. Jama, 310, 2416-2425. 
de Zwaan, M., Enderle, J., Wagner, S., Mühlhans, B., Ditzen, B., Gefeller, O., ... \& Müller, A. (2011). Anxiety and depression in bariatric surgery patients: a prospective, follow-up study using structured clinical interviews. Journal of Affective Disorders, 133, 61-68.

Enders, C. K., \& Bandalos, D. L. (2001). The relative performance of full information maximum likelihood estimation for missing data in structural equation models. Structural Equation Modeling, 8, 430-457.

Engel, S., Mitchell, J.E., de Zwaan, M., \& Steffan, K. J. (2012). Eating disorders and eating problems pre- and postbariatric surgery (pp. 99-110). In: Mitchell, J.E., \& de Zwaan, M. (Eds). Psychosocial assessment and treatment of bariatric surgery patients. Routledge: New York, NY.

Ferguson, C. J. (2009). An effect size primer: A guide for clinicians and researchers. Professional Psychology: Research and Practice, 40, 532-538.

Gardner, B. O., Boccaccini, M. T., Bitting, B. S., \& Edens, J. F. (2015). Personality Assessment Inventory scores as predictors of misconduct, recidivism, and violence: A meta-analytic review. Psychological Assessment, 27, 534-544.

Gerlach, G., Herpertz, S., \& Loeber, S. (2015). Personality traits and obesity: A systematic review. Obesity Reviews, 16, 32-63.

Grimm, K. J., Ram, N., \& Estabrook, R. (2017). Growth modeling: Structural equation and multilevel modeling approaches. The Guilford Press: New York, NY.

Herpertz, S., Kielmann, R., Wolf, A. M., Hebebrand, J., \& Senf, W. (2004). Do psychosocial variables predict weight loss or mental health after obesity surgery? A systematic review. Obesity Research, 12, 1554-1569. 
Kalarchian, M. A., \& Marcus, M. D. (2018). The case for stepped care for weight management after bariatric surgery. Surgery for Obesity and Related Diseases, 14, 112-116.

Kalarchian, M. A., King, W. C., Devlin, M. J., Marcus, M. D., Garcia, L., Chen, J. Y., ... \& Mitchell, J. E. (2016). Psychiatric disorders and weight change in a prospective study of bariatric surgery patients: a 3-year follow-up. Psychosomatic Medicine, 78, 373-381.

Karlsson, J., Taft, C., Sjöström, L., Torgerson, J. S., \& Sullivan, M. (2003). Psychosocial functioning in the obese before and after weight reduction: Construct validity and responsiveness of the Obesity-related Problems scale. International Journal of Obesity, 27, 617-630.

Kline, R. B. (2015). Principles and practice of structural equation modeling. New York, NY: Guilford Press.

Kotov, R., Krueger, R. F., Watson, D., Achenbach, T. M., Althoff, R. R., Bagby, R. M., ... \& Eaton, N. R. (2017). The Hierarchical Taxonomy of Psychopathology (HiTOP): A dimensional alternative to traditional nosologies. Journal of Abnormal Psychology, 126, 454-477.

Livhits, M., Mercado, C., Yermilov, I., Parikh, J. A., Dutson, E., Mehran, A., ... \& Gibbons, M. M. (2012). Preoperative predictors of weight loss following bariatric surgery: Systematic review. Obesity Surgery, 22, 70-89.

Marek, R. J., Ben-Porath, Y. S., \& Heinberg, L. J. (2016). Understanding the role of psychopathology in bariatric surgery outcomes. Obesity Reviews, 17, 126-141.

Marek, R. J., Ben-Porath, Y. S., van Dulmen, M. H., Ashton, K., \& Heinberg, L. J. (2017). Using the presurgical psychological evaluation to predict 5-year weight loss outcomes in bariatric surgery patients. Surgery for Obesity and Related Diseases, 13, 514-521. 
Morey, L. C. (2007). Personality Assessment Inventory (PAI): Professional Manual, 2 nd Edition. Lutz, FL: Psychological Assessment Resources.

Newman, D. A. (2014). Missing data: Five practical guidelines. Organizational Research Methods, 17, 372-411.

O'Leary, A. (1990). Stress, emotion, and human immune function. Psychological Bulletin, 108, 363-382.

Patry, M. W., \& Magaletta, P. R. (2015). Measuring suicidality using the Personality Assessment Inventory: A convergent validity study with federal inmates. Assessment, 22, 36-45.

Peugh, J. L., \& Enders, C. K. (2004). Missing data in educational research: A review of reporting practices and suggestions for improvement. Review of Educational Research, 74, 525556.

Piotrowski, C. (2017). The status of the Personality Assessment Inventory (PAI) in training and practice: Evidence from the emerging literature. SIS Journal of Projective Psychology \& Mental Health, 24, 83-90.

Puzziferri, N., Roshek, T. B., Mayo, H. G., Gallagher, R., Belle, S. H., \& Livingston, E. H. (2014). Long-term follow-up after bariatric surgery: a systematic review. JAMA, 312, 934-942.

R Core Team. (2013). R: A language and environment for statistical computing. Vienna, Austria: R Foundation for Statistical Computing.

Robinson, A. H., Adler, S., Stevens, H. B., Darcy, A. M., Morton, J. M., \& Safer, D. L. (2014). What variables are associated with successful weight loss outcomes for bariatric surgery after 1 year? Surgery for Obesity and Related Diseases, 10, 697-704. 
Rosseel, Y. (2012). lavaan: An R Package for structural equation modeling. Journal of Statistical Software, 48, 1-36. http://www.jstatsoft.org/v48/i02/

Rydén, A., Karlsson, J., Persson, L. O., Sjöström, L., Taft, C., \& Sullivan, M. (2001). Obesityrelated coping and distress and relationship to treatment preference. British Journal of Clinical Psychology, 40, 177-188.

Shen, S. C., Lin, H. Y., Huang, C. K., \& Yen, Y. C. (2016). Adherence to psychiatric follow-up predicts 1-year BMI loss in gastric bypass surgery patients. Obesity Surgery, 26, 810-815.

Slavin-Mulford, J., Sinclair, S. J., Stein, M., Malone, J., Bello, I., \& Blais, M. A. (2012). External validity of the Personality Assessment Inventory (PAI) in a clinical sample. Journal of Personality Assessment, 94, 593-600.

Sogg, S., Lauretti, J., \& West-Smith, L. (2016). Recommendations for the presurgical psychosocial evaluation of bariatric surgery patients. Surgery for Obesity and Related Diseases, 12, 731-749.

Van Hout, G. C., Verschure, S. K., \& Van Heck, G. L. (2005). Psychosocial predictors of success following bariatric surgery. Obesity Surgery, 15, 552-560.

Wasserstein, Schirm, \& Lazar (2019). Moving to a world beyond " $p<0.05$." The American Statistician, 73, 1-19.

Wickham, H. (2016). ggplot2: Elegant Graphics for Data Analysis. Springer-Verlag New York. ISBN 978-3-319-24277-4, http://ggplot2.org.

Wimmelmann, C. L., Dela, F., \& Mortensen, E. L. (2014). Psychological predictors of weight loss after bariatric surgery: a review of the recent research. Obesity Research \& Clinical Practice, 8, e299-e313. 
Table 1

Full-scale PAI T-scores of 2,267 bariatric surgery patients

\begin{tabular}{|c|c|c|c|c|c|c|}
\hline Scale & $M$ & $S D$ & Skewness & Kurtosis & $\alpha$ & MIC \\
\hline \multicolumn{7}{|c|}{ Validity Scales } \\
\hline ICN & 47.88 & 7.92 & 0.58 & -0.06 & & \\
\hline INF & 48.57 & 7.30 & 0.80 & 0.04 & & \\
\hline PIM & 53.24 & 9.70 & -0.38 & 0.09 & & \\
\hline NIM & 47.79 & 5.95 & 2.57 & 9.72 & & \\
\hline \multicolumn{7}{|c|}{ Clinical Scales } \\
\hline SOM & 59.08 & 10.19 & 0.88 & 0.71 & .87 & .21 \\
\hline ANX & 50.40 & 9.36 & 1.07 & 1.48 & .90 & .29 \\
\hline ARD & 48.44 & 9.44 & 0.87 & 0.97 & .76 & .14 \\
\hline DEP & 54.39 & 10.64 & 0.93 & 1.04 & .88 & .26 \\
\hline MAN & 44.43 & 8.50 & 0.65 & 0.39 & .79 & .14 \\
\hline PAR & 47.54 & 8.75 & 0.64 & 0.68 & .82 & .17 \\
\hline SCZ & 44.92 & 8.56 & 1.15 & 2.17 & .81 & .16 \\
\hline BOR & 47.85 & 8.43 & 0.80 & 0.73 & .84 & .18 \\
\hline ANT & 43.92 & 5.92 & 1.24 & 1.80 & .69 & .10 \\
\hline ALC & 44.19 & 4.65 & 2.35 & 9.59 & .53 & .15 \\
\hline DRG & 47.10 & 6.06 & 1.20 & 0.98 & .41 & .05 \\
\hline \multicolumn{7}{|c|}{ Treatment } \\
\hline \multicolumn{7}{|c|}{ Consideration Scales } \\
\hline AGG & 45.74 & 8.47 & 0.98 & 1.23 & .84 & .25 \\
\hline SUI & 45.81 & 5.58 & 3.91 & 24.16 & .72 & .27 \\
\hline NON & 44.21 & 8.85 & 1.62 & 2.75 & .78 & .30 \\
\hline STR & 47.61 & 8.68 & 1.26 & 1.98 & .71 & .27 \\
\hline RXR & 47.51 & 7.51 & -0.08 & -0.18 & .58 & .15 \\
\hline \multicolumn{7}{|c|}{ Interpersonal Scales } \\
\hline DOM & 50.93 & 9.74 & -0.20 & 0.15 & .77 & .22 \\
\hline WRM & 53.32 & 10.11 & -0.52 & 0.09 & .81 & .26 \\
\hline
\end{tabular}

Note. $N=2,267$. ICN = inconsistency, INF = infrequency, $\mathrm{PIM}=$ positive impression management, $\mathrm{NIM}=$ negative impression management, $\mathrm{SOM}=$ somatic complaints, $\mathrm{ANX}=$ anxiety, ARD = anxiety-related disorders, DEP = depression, $\mathrm{MAN}=$ mania, $\mathrm{PAR}=$ paranoia, $\mathrm{SCZ}=$ schizophrenia, $\mathrm{BOR}=$ borderline features, $\mathrm{ANT}=$ antisocial features, $\mathrm{ALC}=$ alcohol problems, $\mathrm{DRG}=$ drug problems, $\mathrm{AGG}=$ aggression, $\mathrm{SUI}=$ suicidal ideation, $\mathrm{NON}=$ nonsupport, $\mathrm{STR}=$ stress, $\mathrm{RXR}=$ treatment rejection, $\mathrm{DOM}=$ dominance, $\mathrm{WRM}=$ warmth. Skewness standard error $=.05$ for all scales. Kurtosis standard error $=.10$ for all scales. Excluding 17 cases greater than or equal to 73 on ICN and 3 cases greater than or equal to 75 on INF (Morey, 2007). Internal consistencies are not provided for validity scales. 
Table 2

PAI Scales Predicting Five-Year Change in BMI Growth Factors Over and Above Covariates

\begin{tabular}{lccccccccccccc}
\hline \multicolumn{10}{c}{} & \multicolumn{10}{c}{ Growth Factors } \\
\hline & \multicolumn{1}{c}{ Intercept } & \multicolumn{1}{c}{ Linear Slope } & \multicolumn{1}{c}{ Quadratic Slope } \\
\hline Model & $b$ & SE & $p$ & $\beta$ & $b$ & $S E$ & $p$ & $\beta$ & $b$ & $S E$ & $p$ & $\beta$ \\
\hline SOM & .066 & .030 & .029 & .07 & -.033 & .014 & .019 & -.08 & .010 & .003 & $<.001$ & .09 \\
ANX & .057 & .032 & .076 & .05 & -.015 & .013 & .246 & -.04 & .001 & .002 & .520 & .02 \\
ARD & .120 & .033 & $<.001$ & .12 & -.032 & .014 & .022 & -.08 & .003 & .003 & .182 & .03 \\
DEP & .060 & .027 & .024 & .06 & -.028 & .013 & .024 & -.07 & .006 & .002 & .016 & .06 \\
MAN & .052 & .034 & .132 & .05 & -.010 & .019 & .602 & -.02 & .001 & .003 & .727 & .01 \\
PAR & .080 & .034 & .017 & .07 & -.008 & .011 & .479 & -.03 & .000 & .002 & .987 & .00 \\
SCZ & .066 & .035 & .062 & .06 & -.021 & .018 & .231 & -.04 & .002 & .003 & .520 & .02 \\
BOR & .100 & .035 & .005 & .09 & -.031 & .017 & .066 & -.06 & .004 & .003 & .186 & .03 \\
ANT & .054 & .051 & .288 & .03 & .002 & .025 & .923 & .00 & .001 & .005 & .895 & .00 \\
ALC & .140 & .056 & .012 & .07 & -.026 & .029 & .373 & -.03 & .000 & .005 & .996 & .00 \\
DRG & .129 & .049 & .008 & .08 & -.001 & .023 & .961 & .00 & -.004 & .004 & .263 & -.03 \\
AGG & .031 & .033 & .345 & .03 & .007 & .017 & .690 & .01 & -.002 & .003 & .453 & -.02 \\
SUI & .048 & .051 & .352 & .03 & .012 & .023 & .601 & .02 & -.001 & .004 & .776 & -.01 \\
STR & .118 & .034 & .001 & .10 & -.054 & .016 & .001 & -.11 & .011 & .003 & $<.001$ & .10 \\
NON & .094 & .032 & .003 & .08 & -.034 & .017 & .040 & -.07 & .005 & .003 & .126 & .04 \\
RXR & -.178 & .038 & $<.001$ & -.14 & .068 & .018 & $<.001$ & .12 & -.012 & .003 & $<.001$ & -.09 \\
DOM & -.004 & .030 & .899 & .00 & .029 & .014 & .036 & .07 & -.006 & .003 & .018 & -.06 \\
WRM & -.020 & .027 & .450 & -.02 & .017 & .012 & .158 & .04 & -.002 & .002 & .473 & -.02 \\
\hline Note SOM
\end{tabular}

Note. $\mathrm{SOM}=$ somatic complaints, ANX = anxiety, ARD = anxiety-related disorders, DEP = depression, $\mathrm{MAN}=$ mania, $\mathrm{PAR}=$ paranoia, $\mathrm{SCZ}=$ schizophrenia, $\mathrm{BOR}=$ borderline, $\mathrm{ANT}=$ antisocial, $\mathrm{ALC}=$ alcohol problems, $\mathrm{DRG}=$ drug problems, $\mathrm{AGG}=$ aggression, $\mathrm{SUI}=$ suicidal, $\mathrm{STR}=$ stress, $\mathrm{NON}=$ nonsupport, $\mathrm{RXR}=$ treatment rejection, $\mathrm{DOM}=$ dominance, $\mathrm{WRM}=$ warmth. Each row is a separate statistical model. 
Table 3. PAI Subscales Predicting Five-Year Change in BMI Growth Factors Over and Above Covariates.

\begin{tabular}{|c|c|c|c|c|c|c|c|c|c|c|c|c|}
\hline \multirow[b]{2}{*}{ Model } & \multicolumn{4}{|c|}{ Intercept } & \multicolumn{4}{|c|}{ Linear Slope } & \multicolumn{4}{|c|}{ Quadratic Slope } \\
\hline & $b$ & $S E$ & $p$ & $\beta$ & $b$ & $S E$ & $p$ & $\beta$ & $b$ & $S E$ & $p$ & $\beta$ \\
\hline SOM_C & .239 & .089 & .007 & .09 & -.113 & .041 & .006 & -.09 & .029 & .007 & $<.001$ & .10 \\
\hline SOM_S & .040 & .028 & .161 & .04 & -.035 & .013 & .007 & -.08 & .010 & .002 & $<.001$ & .10 \\
\hline SOM_H & .048 & .031 & .114 & .05 & -.010 & .014 & .464 & -.02 & .005 & .003 & .076 & .04 \\
\hline ANX_C & .058 & .030 & .055 & .06 & -.013 & .013 & .325 & -.03 & .000 & .002 & .898 & .00 \\
\hline ANX_A & .051 & .032 & .110 & .05 & -.018 & .014 & .194 & -.04 & .002 & .002 & .316 & .02 \\
\hline ANX_P & .040 & .034 & .233 & .04 & -.009 & .015 & .550 & -.02 & .002 & .003 & .562 & .01 \\
\hline ARD_O & .049 & .029 & .098 & .05 & .003 & .013 & .805 & .01 & -.004 & .002 & .083 & -.04 \\
\hline ARD_P & .111 & .034 & .001 & .11 & -.050 & .015 & .001 & -.11 & .009 & .003 & $<.001$ & .09 \\
\hline ARD_T & .091 & .034 & .007 & .09 & -.023 & .016 & .140 & -.05 & .002 & .003 & .501 & .02 \\
\hline DEP_C & .198 & .087 & .024 & .07 & -.089 & .041 & .030 & -.07 & .015 & .007 & .046 & .05 \\
\hline DEP_A & .052 & .026 & .045 & .06 & -.015 & .011 & .184 & -.04 & .002 & .002 & .214 & .03 \\
\hline DEP_P & .040 & .026 & .130 & .05 & -.028 & .013 & .033 & -.07 & .006 & .002 & .010 & .07 \\
\hline MAN_A & .018 & .033 & .585 & .02 & -.014 & .016 & .385 & -.03 & .001 & .003 & .689 & .01 \\
\hline MAN_G & .022 & .033 & .502 & .02 & .010 & .015 & .490 & .02 & .000 & .003 & .940 & .00 \\
\hline MAN_I & .060 & .034 & .076 & .06 & -.017 & .016 & .287 & -.04 & .001 & .003 & .684 & .01 \\
\hline PAR_H & .063 & .031 & .044 & .06 & -.012 & .015 & .431 & -.03 & .001 & .003 & .776 & .01 \\
\hline PAR_P & .104 & .045 & .022 & .07 & -.008 & .022 & .705 & -.01 & -.001 & .004 & .816 & -.01 \\
\hline PAR_R & .051 & .029 & .083 & .05 & .000 & .015 & .988 & .00 & .000 & .003 & .890 & .00 \\
\hline SCZ_P & .407 & .153 & .008 & .08 & -.083 & .061 & .172 & -.05 & .009 & .011 & .386 & .02 \\
\hline SCZ_S & .042 & .030 & .158 & .04 & -.022 & .015 & .143 & -.05 & .003 & .003 & .339 & .02 \\
\hline SCZ_T & .014 & .032 & .658 & .01 & .000 & .016 & .993 & .00 & -.001 & .003 & .761 & -.01 \\
\hline BOR_A & .048 & .033 & .149 & .04 & -.020 & .016 & .198 & -.04 & .003 & .003 & .347 & .02 \\
\hline BOR_I & .105 & .035 & .003 & .09 & -.038 & .014 & .009 & -.09 & .006 & .002 & .020 & .06 \\
\hline BOR_N & .089 & .030 & .003 & .09 & -.010 & .014 & .475 & -.02 & .000 & .002 & .860 & .00 \\
\hline BOR_S & .038 & .038 & .311 & .03 & -.022 & .020 & .258 & -.04 & .004 & .004 & .235 & .03 \\
\hline ANT_A & .040 & .045 & .372 & .03 & .008 & .021 & .695 & .01 & .001 & .004 & .746 & .01 \\
\hline ANT_E & -.044 & .054 & .414 & -.03 & .034 & .024 & .162 & .05 & -.005 & .004 & .219 & -.03 \\
\hline ANT_S & .164 & .121 & .175 & .04 & -.058 & .051 & .256 & -.04 & .004 & .009 & .670 & .01 \\
\hline AGG_A & .040 & .031 & .196 & .04 & .002 & .015 & .883 & .01 & -.002 & .003 & .439 & -.02 \\
\hline AGG_V & .001 & .031 & .971 & .00 & .015 & .016 & .354 & .03 & -.003 & .003 & .326 & -.03 \\
\hline AGG_P & .052 & .045 & .255 & .03 & -.017 & .022 & .451 & -.02 & .003 & .004 & .539 & .02 \\
\hline
\end{tabular}

Note. $\mathrm{SOM}-\mathrm{C}=$ conversion, $\mathrm{SOM}-\mathrm{S}=$ somatization, $\mathrm{SOM}-\mathrm{H}=$ health concerns, $\mathrm{ANX}-\mathrm{C}=$ cognitive, ANX-A = affective, ANX-P = physiological, ARD-O = obsessive-compulsive, ARD$\mathrm{P}=$ phobias, $\mathrm{ARD}-\mathrm{T}=$ traumatic stress, $\mathrm{DEP}-\mathrm{C}=$ cognitive, $\mathrm{DEP}-\mathrm{A}=$ affective, $\mathrm{DEP}-\mathrm{P}=$ physiological, MAN-A = activity level, MAN-G = grandiosity, MAN-I = irritability, PAR-H = hypervigilance, $\mathrm{PAR}-\mathrm{P}=$ persecution, $\mathrm{PAR}-\mathrm{R}=$ resentment, $\mathrm{SCZ}-\mathrm{P}=$ psychotic experiences, 
SCZ-S = social detachment, SCZ-T = thought disorder, BOR-A = affective instability, BOR-I = identity problems, BOR-N = negative relationships, BOR-S = self-harm, ANT-A = antisocial behaviors, ANT-E = egocentricity, ANT-S = stimulus-seeking, AGG-A = aggressive attitude, AGG-V = verbal aggression, AGG-P = physical aggression. Each row is a separate statistical model. 


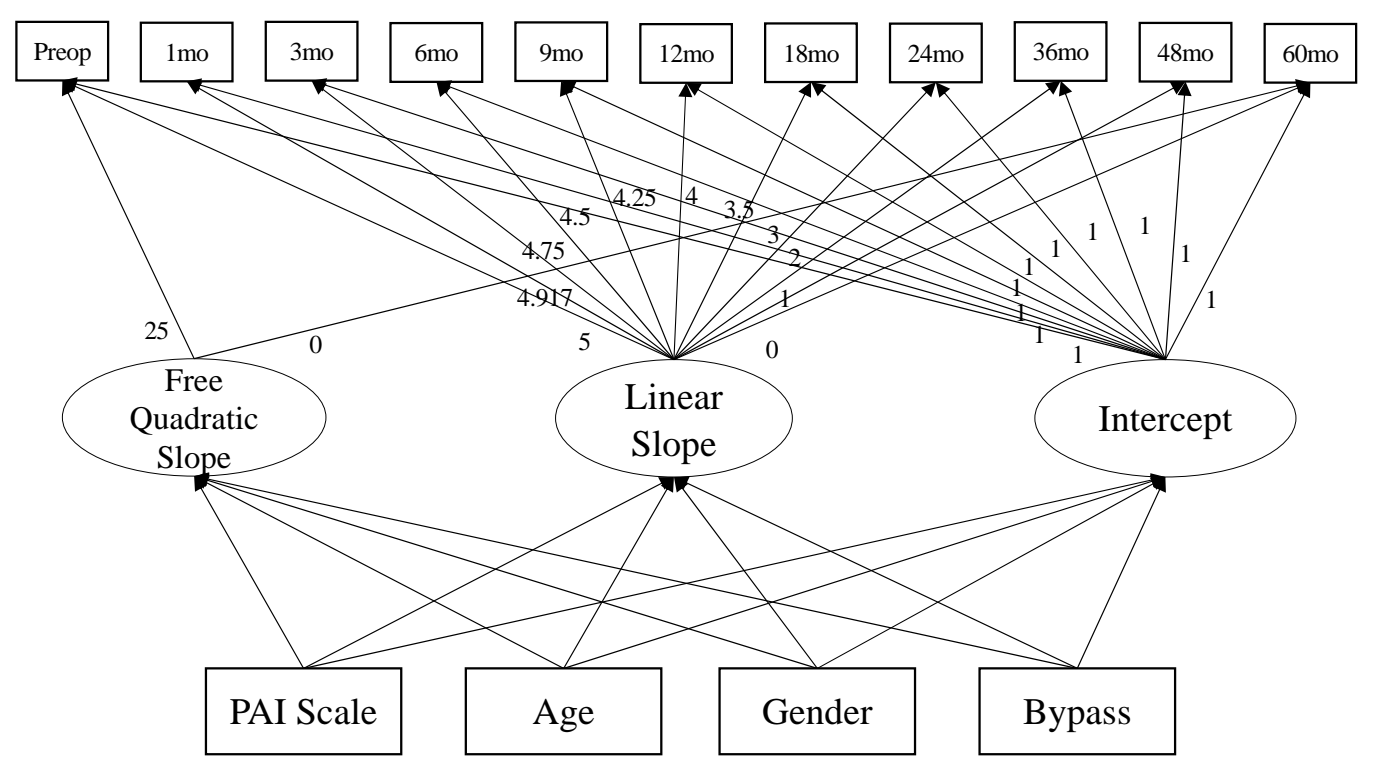

Figure 1. Free quadratic latent growth curve prediction model. 